\title{
SPIRULINA ACCEPTABILITY TRIALS IN RATS. A STUDY FOR THE "MELISSA" LIFE-SUPPORT SYSTEM
}

\author{
N. Tranquille, ${ }^{*}$ J. J. Emeis, ${ }^{*}$ D. de Chambure, ${ }^{* *}$ R. Binot** and \\ C. Tamponnet** \\ * Gaubius Laboratory, IVVO-TNO, P.O. Box 430, 2300 AK Leiden, \\ The Netherlands \\ ** Thermal Control and Life Support Division, ESA-ESTEC, P.O. Box 299, \\ 2200 AG Noordwijk, The Netherlands
}

\begin{abstract}
Groups of five rats were fed for sixteen weeks a slightly deficient diet, supplemented with $0-40 \%$ of a dried preparation of the blue-green alga Spirulina as a protein source. Control groups were fed a normal rat diet. No significant differences between groups were found in food intake, growth rate or carbon dioxide production. All animals remained apparently healthy, and had similar organ weights. The study suggests that Spirulina may be used as a protein source in rat diets.
\end{abstract}

\section{INTRODUCTION}

Spirulina, a microscopic blue-green alga $/ 1,2 /$, has been consumed by man since ancient times. This alga has a very high protein content (about $70 \%$ of its dry matter) and is therefore very nutritious. The two main species of Spirulina: Spirulina platensis found in the Kanem regio of Chad /3/, and Spirulina maxima found around Lake Texcoco in Mexico /4/, are being cultivated artificially and sold as a dry powder. In contrast to its high protein content Spirulina contains a lower concentration of nucleic acids (4\% of dry matter) than is found in most high-protein containing bacteria $12,5 /$. In addition, Spirulina contains more than adequate amounts of vitamins and unsaturated fatty acids, and its amino-acid spectrum is normal, except for a slight deficiency in the sulphur amino acids, including methionine $15-7 /$.

The most detailed studies on the nutritional value of Spirulina have been conducted on rodents, mainly rats $18-15 /$, though this has also been studied in poultry and pigs. The rodent experiments covered a variety of parameters, from the basic nutritional value of Spirulina platensis /8/ and Spirulina maxima $19,10 /$ to vitamin bioavailability from Spirulina in rats $/ 11,12 /$, and the improvement of dietary hyperlipidaemia $/ 13 /$. The nutritional value of Spirulina as a food source has led scientists to include Spirulina as a dietary component of a biological life support system $/ 16 /$.

MELISSA is a model of a future ecological life support system /17/ consisting of four compartments, one being a central food and oxygen reprocessing compartment growing Spirulina, which is the main source of food in this closed loop life support model. The studies reported in this paper investigated the food acceptability of Spirulina and its suitability as a component of the consumer diet in the MELISSA ecosystem model. The aim of this study was to determine whether eating Spirulina had any advantages over a slightly deficient diet, and whether Spirulina showed any toxic effects when eaten daily by rats for a sixteen week period.

\section{MATERIALS AND METHODS}

Animals Thirty male Wistar rats (six weeks of age, and weighing about $150 \mathrm{~g}$ ) were obtained from our Institute's breeding colony. The animals were housed individually, in macrolon cages, under standard conditions (12 hours light/12 hours dark; temperature $21-22^{\circ} \mathrm{C}$; humidity $60-70 \%$ ). The animals were assessed three times a week, when their body weight and their food and water intake were measured, and their general behaviour was assessed. Food and acidified drinking water were provided ad libitum. 
Diets The following diets were used:

a. $\quad$ standard complete rat diet (pelleted control diet)

b. $\quad$ standard complete rat diet (flour in agar, see below; control diet)

c. deficient diet (flour in agar)

d. deficient diet $/ 5 \%$ Spirulina (flour in agar)

e. deficient diet/10\% Spirulina (flour in agar)

t. $\quad$ deficient diet $/ 40 \%$ Spirulina (tlour in agar).

Diet a was used as provided by the manufacturer (Hope Farms, Woerden, the Netherlands). Diets b-f were prepared as follows: $2 \%(\mathrm{w} / \mathrm{v})$ agar-agar was dissolved in water by heating. The agar solution was then cooled while stirring, added to the other dietary components (1 part of agar solution to 2 parts of the Spirulina/flour mix) and well mixed till it formed a firm dough. This dough was shaped into flat cakes, cut into cubes and kept frozen at $-20^{\circ} \mathrm{C}$ until use. Spirulina was obtained as a dry powder from Pharmafood B.V., Putten, the Netherlands. The other dietary components (basic diet, cerelose and complete diet) were obtained as flour from Hope Farms. The basic diet had been formulated in such a way that the deficient diet $\underline{c}$ had an overall slight deficiency in all nutrients. The detailed composition of the diets is available from the authors upon request.

Experimental design The animals were randomly divided into six groups of five rats.

Four groups were fed either diet $\underline{a}, \underline{b}, \underline{c}$ or $\underline{d}$ for sixteen weeks. A fifth group was fed diet $\underline{e}$ for eight weeks, and then changed to diet $\underline{\mathrm{f}}$ for another eight weeks, while group six was fed diet $\underline{\mathbf{f}}$ for eight weeks, and then changed to diet $\underline{e}$ for eight weeks.

At the end of the sixteen week period, the rats were bled under Nembutal anaesthesia, and their organ weights were determined. Carbon dioxide production was determined in week sixteen.

\section{RESULTS}

Growth curves Figure 1 shows graphically the growth of the rats on the different diets (week 16 has not been included as the rats were moved around and this caused uncontrolled weight changes due to the stress and changes of environment.) The data for each growth point is an average of the five values from the five rats in each group. The initial average daily weight increase per rat was calculated as $\mathbf{5} \mathbf{g}$ a day and this gradually decreased over the next 15 weeks to an average daily weight increase of $1 \mathrm{~g}$ a day. It must be noted that after week 8 the rats on the $10 \%$ Spirulina Diet and the $40 \%$ Spirulina Diet were switched. No major differences in growth were noted between the different groups.

Fond consumption Figure 2 shows the daily food consumption per rat. Each data point presented is an average of the daily food intake of five rats per group per week over the experimental period. (Weeks 6-10 and week 16 have not been shown as during these periods their feeding habits were disrupted.) The rats on the pelleted control diet appear to have consumed less food than the other rats on the 5 prepared diets. However, the water content in the 5 prepared diets is a third of the total weight. When this is taken into consideration all groups consumed about the same amount of dry weight of food.

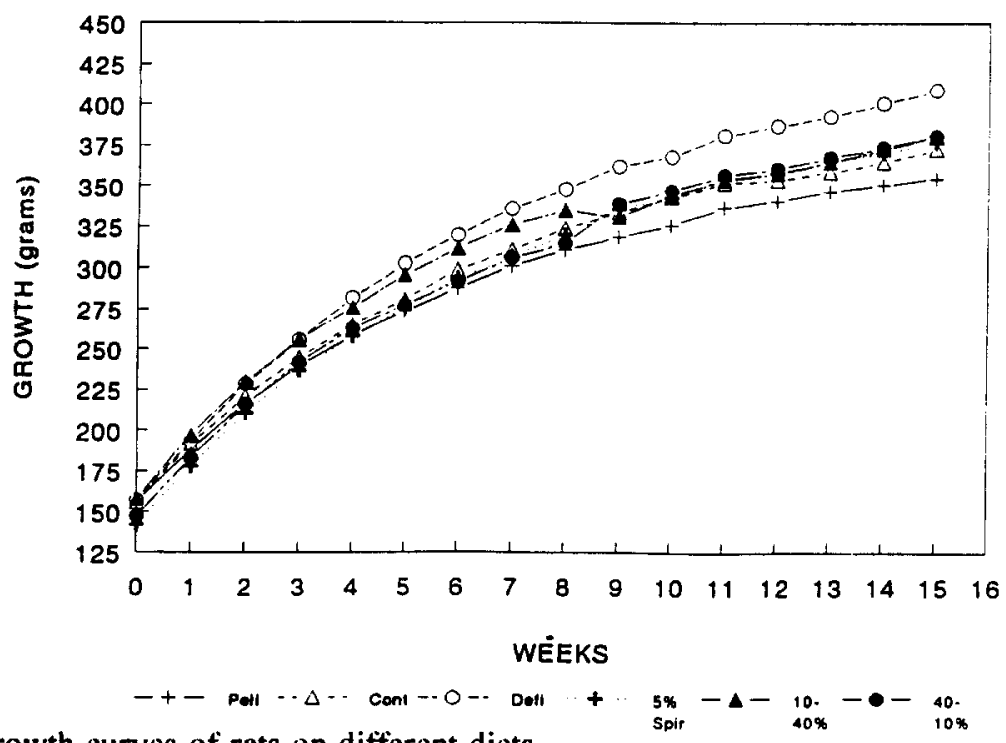

Fig. 1. Growth curves of rats on different diets. 


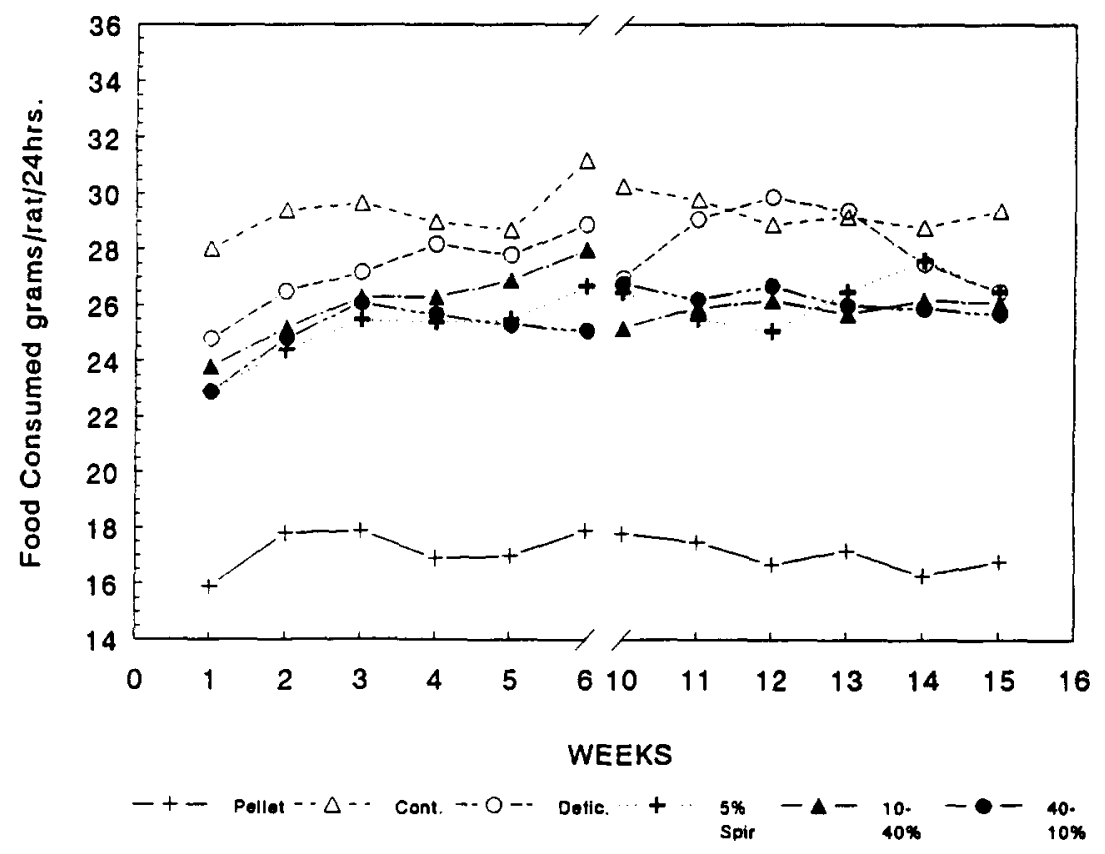

Fig. 2. Food consumption of rats on different diets.

General health of the rats At the conclusion of the experiment the animals' general health was assessed. All the animals had healthy looking teeth, skin and internal organs. All the major organs were inspected and weighed. Table 1 below shows the weight of the major organs of the rats on the different diets and the values all fall within the normal documented values (except for the liver weights where a significant difference ( $p<0.05$ by ANOVA) between the groups was noted).

\section{TABLE 1}

\begin{tabular}{lcccc}
\hline Diet & $\begin{array}{c}\text { Liver } \\
(\mathrm{g})\end{array}$ & $\begin{array}{c}\text { Kidneys } \\
(\mathrm{g})\end{array}$ & $\begin{array}{c}\text { Lungs } \\
(\mathrm{g})\end{array}$ & $\begin{array}{c}\text { Heart } \\
(\mathrm{g})\end{array}$ \\
\hline Pelleted control diet & $8.55 \pm 0.05$ & $2.17 \pm 0.13$ & $1.34 \pm 0.27$ & $1.04 \pm 0.10$ \\
Control diet & $9.40 \pm 0.49$ & $2.36 \pm 0.05$ & $1.39 \pm 0.14$ & $1.06 \pm 0.04$ \\
Deficient diet & $10.21 \pm 1.12$ & $2.44 \pm 0.18$ & $1.44 \pm 0.16$ & $1.11 \pm 0.05$ \\
5\% Spirulina diet & $7.86 \pm 0.47$ & $2.14 \pm 0.23$ & $1.41 \pm 0.22$ & $1.11 \pm 0.08$ \\
10\% Spirulina diet & $9.10 \pm 0.74$ & $2.12 \pm 0.12$ & $1.38 \pm 0.13$ & $1.05 \pm 0.11$ \\
40\% Spirulina diet & $9.07 \pm 1.96$ & $2.33 \pm 0.39$ & $1.46 \pm 0.19$ & $1.05 \pm 0.07$ \\
\hline
\end{tabular}

Carbon Dioxide Production Experiments It was considered of interest to try and measure the amounts of carbon dioxide produced, and oxygen consumed, by the rats on the different diets. This was done using on-line mass spectrometric analysis of air from a controlled closed chamber (1 cubic meter) in which each rat was placed for 30 minutes. For oxygen consumption differences between measurements were too small to be useful. However, carbon dioxide production could be measured reliably over the 30 minute period; these changes are shown in Figure 3. The data is based on four rats for each dietary group. The results show that the rats on the $40 \%$ Spirulina diet produced less carbon dioxide than rats from the other groups, though these differences were not found to be statistically significant.

\section{DISCUSSION}

The results of our work clearly show the nutritional value of Spirulina as part of the protein content of a diet. Using young male rats enabled us to follow their growth pattern and, as can be seen in Figure 1 , the rats on the Spirulina diets followed a normal growth pattern. This has also been observed in similar studies by Maranesi et al. /10/ and Chamorro et al. /14/. The food consumption of the animals on the Spirulina diet was slightly lower (Figure 2) compared to the Control diet; however, this difference was not significant. A similar observation was also noted by Chamorro et al. /14/ in their feeding studies. 


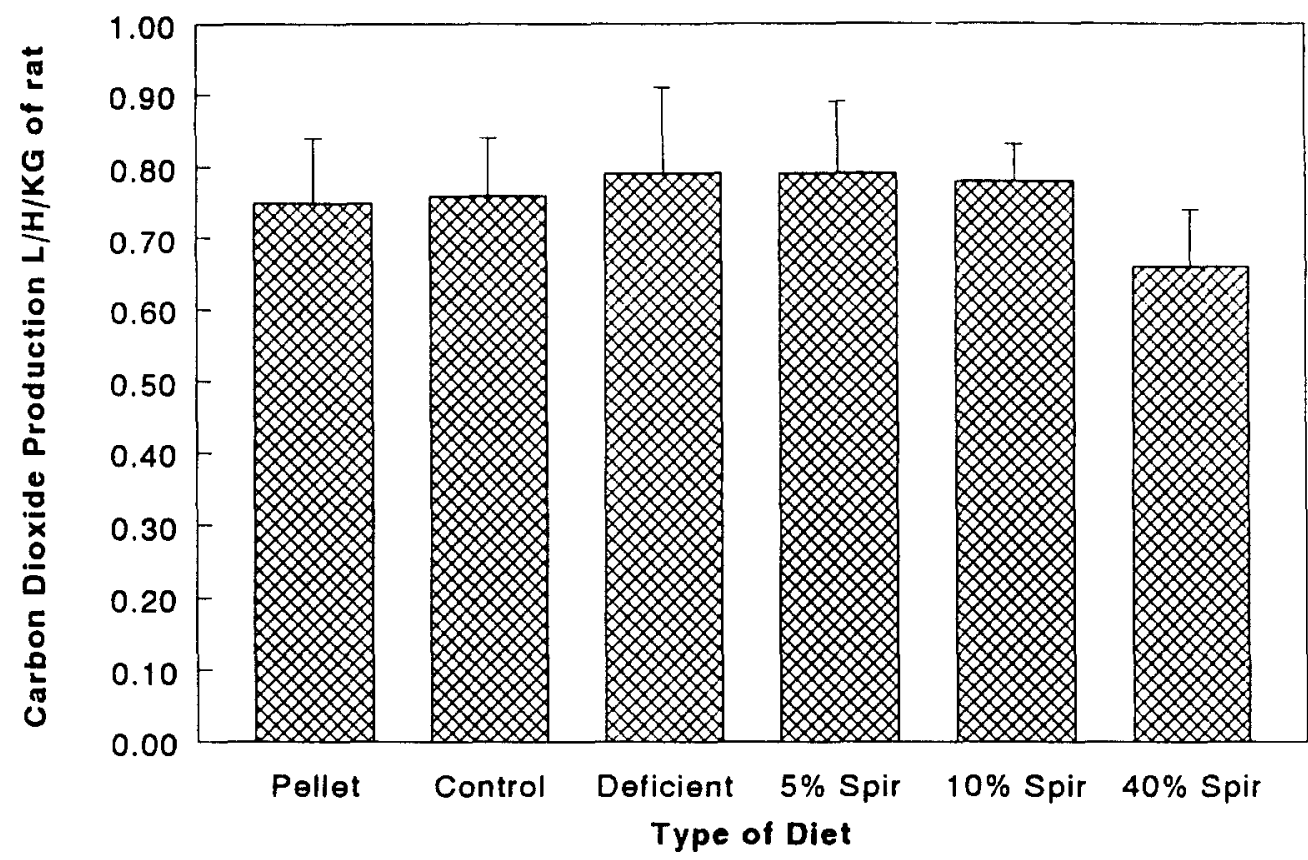

Fig. 3 Carbon dioxide production.

As other scientists have observed $/ 10,14 /$, no differences as a result of the different diets were seen in the lungs and heart weights from the different rats. The kidneys weights were also found to be normal. Creatinine levels were normal and below the level for kidney disfunction (not shown). A series of other biochemical, physiological and metabolic variables were also measured during and at the end of this study. These will be presented and discussed elsewhere.

To briefly summarize, the present data and other short-term studies (e.g. /10,14/) favourably supports the theory that Spirulina may be used as a component of the diet in a future ecological life support system such as MELISSA.

\section{REFERENCES}

1. O. Ciferri, Microbiol. Rev. 47, 551-578 (1983).

2. O. Ciferri and O. Tiboni, Ann. Rev. Microbiol. 39, 503-526 (1985).

3. F. Delpeuch, A. Joseph and C. Cavlier, Ann. Nutr. Aliment. 29, 497-516 (1975).

4. G. Clement, Ann. Nutr. Aliment. 29, 477-488 (1975).

5. G. Clement, C. Giddey and R. Menzi, J. Sci. Food Agric. 18, 494-501 (1967).

6. M. Vermorel, G. Toullec, D. Dumond and R. Pion, Ann. Nutr. Aliment. 29, 535-552 (1975).

7. P.T. Omstedt, A. Von der Decken, G. Hedenskog and H. Mogren, J. Sci. Food Agric. 24, 11031113 (1973).

8. D.L. Narasimha, G.S. Venkataraman, S.K. Duggal and B.O. Eggum, J. Sci. Food Agric. 33, 456460 (1982).

9. I. Tejada de Hernandez and A.S. Shimada, Arch. Latinoam. Nutr. 28, 196-207 (1978).

10. M. Maranesi, V. Barzanti, G. Carenini and P. Gentili, Acta Vitaminol. Enzymol. 6, 295-304 (1984).

11. H. Van den Berg, L. Brandsen and B.J. Sinkeldam, J. Nutr. Biochem. 2, 314-318 (1991).

12. G.V. Mitchell, E. Grundel, M. Jenkins and S.R. Blakely, J. Nutr. 120, 1235-1240 (1990).

13. K. Iwata, T. Inayama and T. Kato, J. Nutr. Sci. Vitaminol. 36, 165-171 (1990).

14. G.A. Chamorro, G. Herrera, M. Salazar, S. Salazar and V. Ulloa, J. Pharm. Belg. 43, 29-36 (1988).

15. Y. Yoshino, Y. Hirai, H. Takahashi, N. Yamamoto and N. Yamazaki, Jap. J. Nutr. 38, 221-225 (1980).

16. A.A. Antonian, I.A. Abakumova, G.I. Meleshko and T.F. Vlasova, Kosm. Biol. Aviakosm. Med. $19,65-69$ (1985).

17. M. Mergeay, W. Verstraete, G. Dubertret, M. Lefort-Tran, C. Chipaux and R.A. Binot, in: Proc. 3rd Eur. Symp. on Space Thermal Control \& Life Support Systems, Noordwijk, the Netherlands, 1988, ESA SP-288 Dec 1988. p. 65-68. 